\title{
A Causal Channel Model for the Terahertz Band
}

\author{
Kazuhiro Tsujimura $^{(0)}$, Kenta Umebayashi ${ }^{(0)}$, Member, IEEE, Joonas Kokkoniemi ${ }^{\circledR}$, Member, IEEE, \\ Janne Lehtomäki, Member, IEEE, and Yasuo Suzuki, Member, IEEE
}

\begin{abstract}
Impulse response is vital for wireless communication analysis and modeling. This paper considers the impulse response of the terahertz band ( $\mathrm{THz}$ band: 0.1-10 $\mathrm{THz}$ ) for short range $(1-100 \mathrm{~cm})$ wireless communication. Earlier works derived the impulse response from transmittance by assuming a linear phase, which corresponds to a line-of-sight $(\mathrm{LoS})$ propagation delay to a receiver. However, the linear phase leads to a symmetric impulse response before and after the LoS propagation delay. Physically, it is impossible for a signal to arrive before the LoS propagation delay since this violates causality. To address this issue, this study derives a phase function leading to an impulse response that satisfies causality. The validity of the derived model is verified with experimental THz band measurements (up to $2 \mathrm{THz}$ ), which show excellent agreement with the results predicted by the theory. From the impulse response, coherence bandwidth is found for both the entire THz band and its subbands. The results show significant variations in the coherence bandwidth as a function of the center frequency. Knowledge of these variations supports selection of the proper center frequency for wireless communications in the $\mathbf{T H z}$ band.
\end{abstract}

Index Terms-Causality, channel models, impulse response, molecular absorption, terahertz band.

\section{INTRODUCTION}

$\mathbf{T}$ $\mathrm{HE}$ demand for ever high data rates in wireless communication systems is increasing exponentially, and it is predicted that they will reach $10 \mathrm{~Gb} / \mathrm{s}$ for wireless local area networks [1], [2]. The terahertz band (THz band: $0.1-10.0 \mathrm{THz}$ ) is an encouraging candidate for ultra-high-speed communication [2], [3]. Traditional wireless communication systems operate at significantly lower frequencies, such as in the ultra high frequency band (UHF: $300 \mathrm{MHz}-3 \mathrm{GHz}$ ), where extensive investigations on channel modeling have been made [4]. In contrast to the UHF band, the effect of molecular absorption needs to be considered in the $\mathrm{THz}$ band [5].

Manuscript received August 18, 2017; accepted October 24, 2017. Date of publication November 28, 2017; date of current version January 9, 2018. The work of J. Kokkoniemi and J. Lehtomäki was supported in part by the Academy of Finland, in part by the Infotech Oulu Doctoral Program, and in part by the TERRANOVA project funded by Horizon 2020, European Union's Framework Programme for Research and Innovation under Grant 761794. (Corresponding author: Kazuhiro Tsujimura.)

$\mathrm{K}$. Tsujimura and K. Umebayashi are with the Department of Electrical and Electronic Engineering, Tokyo University of Agriculture and Technology, Tokyo, Japan (e-mail: kaz-260@st.go.tuat.ac.jp; ume_k@cc.tuat.ac.jp) .

J. Kokkoniemi and J. Lehtomäki are with the Centre for Wireless Communications, University of Oulu, Oulu 90014, Finland (e-mail: Joonas. Kokkoniemi@oulu.fi; janne.lehtomaki@oulu.fi).

Y. Suzuki was with the Department of Electrical and Electronic Engineering, Tokyo University of Agriculture and Technology, Tokyo, Japan (e-mail: ysuzuki@pf7.so-net.ne.jp)

Color versions of one or more of the figures in this paper are available online at http://ieeexplore.ieee.org.

Digital Object Identifier 10.1109/TTHZ.2017.2771476
Molecular absorption and its impact on transmittance of the channel were studied in [5]. Transmittance gives information about the fraction of the radiation capable of propagating through the channel and is modeled with the Beer-Lambert law. Thus, the Beer-Lambert law directly gives the molecular absorption loss in the channel [5]. Molecular absorption depends on the composition of a medium, relative humidity (RH), pressure, and temperature, and it causes frequency-selective fading for wideband signals.

To develop wireless communication techniques for the $\mathrm{THz}$ band, the impulse response is required. One reasonable approach to obtain impulse response is to use transmittance with only amplitude information and no phase information. In [3], [6], and [7], a linear phase was added to the transmittance and the impulse response was obtained by inverse Fourier transform (IFT). However, the impulse response based on a linear phase, or with no phase shift at all, does not satisfy causality. In [8], a method to obtain the received signal in the time domain was introduced. However, the impulse response as a time-domain channel model was not investigated, and the maximum frequency was around $2 \mathrm{THz}$. In addition, the distance considered in [8] was hundreds of meters. In contrast, we focus on the full terahertz band (0.110.0 THz) and consider communication distances suitable for short-range wireless communication (less than $1 \mathrm{~m}$ ).

In [9], time domain channel model for line-of-sight path is derived from frequency response with spreading loss, and molecular absorption. Unlike previous studies [3], [6], [7] that use linear phase criterion, we obtain the phase component of the impulse response based on minimum phase, leading to a physically valid causal impulse response. A similar approach has been used in the field of spectroscopy [10]. More specifically, a minimum phase is obtained from the amplitude response of the channel by calculating the refractive index of the medium. The refractive index is obtained as a Hilbert transform from the absorption coefficient of the Beer-Lambert law. To the best of our knowledge, we are the first to utilize this method to obtain the impulse response with minimum phase for terahertz communication channels. We show the validity of the derived impulse response by comparing its results to experimental data.

In addition, we evaluate the coherence bandwidth with a given center frequency in a limited frequency band (LFB) scenario. In this scenario, bandpass filters (BPFs) are implemented at both the transmitting and the receiving ends. Frequencies with deep fading in the UHF band depend on a multipath environment and are typically random. In the $\mathrm{THz}$ band, the deep fades are caused by not only a multipath environment but also molecular absorption. Molecular absorption occurs in the resonance 
TABLE I

Key QuANTITIES USED IN THIS PAPER

\begin{tabular}{lcc}
\hline \hline Quantity & Symbol & Unit \\
\hline Absorption coefficient & $k_{a}$ & $\mathrm{~cm}^{-1}$ \\
Distance of $m$ th path & $z_{m}$ & $\mathrm{~cm}$ \\
Frequency & $f$ & $\mathrm{~Hz}$ \\
Pressure & $p$ & $\mathrm{hPa}$ \\
Temperature & $T$ & $\mathrm{~K}$ \\
Speed of light & $c$ & $\mathrm{~cm} / \mathrm{s}$ \\
Angle of incident wave of $m$ th path & $\theta_{m}$ & $\mathrm{deg}$ \\
Rough surface height standard derivation & $\sigma$ & $\mathrm{cm}$ \\
\hline \hline
\end{tabular}

frequencies of molecules in the transmission medium and is an approximately deterministic phenomenon [11]. Therefore, the impact of molecular absorption on the coherence bandwidth is deterministic.

We further derive channel power loss, which represents power loss with a coherence bandwidth constraint determined by a target-flat frequency bandwidth. The channel power loss is useful for designing wireless communication techniques for the $\mathrm{THz}$ band.

The rest of this paper is organized as follows. Section II briefly describes the utilized propagation model for the $\mathrm{THz}$ band. In Section III, we derive the impulse response with the minimum phase. Section IV shows some numerical examples and studies the coherence bandwidth and the power loss of the $\mathrm{THz}$ channels. Section V concludes this paper.

\section{Channel Model}

We assume a multipath channel model in which there is a LoS path and reflected paths between a transmitter and a receiver. Spreading loss and molecular absorption are considered as the dominant loss mechanisms in free space. Reflected paths are derived from Kirchhoff's theory [2], [3]. Antenna characteristics are not considered in the model. Key variables and constants used in this paper are summarized in Table I.

\section{A. Channel Model in the Frequency Domain}

The multipath channel consists of the LoS path and the reflected path. The frequency response of the multipath channel $H(f)$ is defined by

$$
H(f)=\sum_{m=1}^{M} H_{m}\left(f, z_{m}\right)
$$

where $f, z_{m}$, and $H_{m}\left(f, z_{m}\right)$ are the frequency, the distance of the $m$ th path, and the frequency response of the $m$ th path, respectively. The frequency response $H_{1}\left(f, z_{1}\right)=H_{1, \text { los }}\left(f, z_{1}\right)$ corresponds to the LoS path and $H_{m}\left(f, z_{m}\right)=H_{m \text {,refl }}\left(f, z_{m}\right)$ for $m \neq 1$ corresponds to the $m$ th reflected path.

We can describe $H_{m}\left(f, z_{m}\right)$ as follows:

$$
H_{m}\left(f, z_{m}\right)=\left|H_{m}\left(f, z_{m}\right)\right| \exp \left(j \phi_{m}\left(f, z_{m}\right)\right)
$$

where $\phi_{m}$ is the phase component of the $m$ th path. Conventional phase derivation and our proposed phase derivation will be shown in Section III.

1) LoS path: The propagation distance between the transmitter and the receiver is set to $z_{1} \mathrm{~cm}$. The transmittance $\left|H_{1, \text { los }}\left(f, z_{1}\right)\right|^{2}$ is defined as

$$
\left|H_{1, \operatorname{los}}\left(f, z_{1}\right)\right|^{2}=\frac{P_{\text {los }}\left(f, z_{1}\right)}{P\left(f, z_{1}=0\right)}
$$

where $f$ is the frequency, $P\left(f, z_{1}=0\right)$ represents the transmitted signal power, $P_{\text {los }}\left(f, z_{1}\right)$ is the received signal power, and $H_{1, \operatorname{los}}\left(f, z_{1}\right)$ is the frequency response. As can be seen in (3), the transmittance contains the amplitude component of the frequency response, but not the phase component. By considering the spreading loss and the molecular absorption loss, the transmittance (3) can be calculated as [5]

$$
\left|H_{1, \operatorname{los}}\left(f, z_{1}\right)\right|^{2}=\left[A_{\text {abs }}\left(f, z_{1}\right) \times A_{\text {spread }}\left(z_{1}\right)\right]^{-1}
$$

where $A_{\text {spread }}\left(z_{1}\right)$ is the spreading loss and $A_{\text {abs }}\left(f, z_{1}\right)$ is the molecular absorption loss. The spreading loss in the LoS path for an ideal isotropic transmitter is $A_{\text {spread }}\left(z_{1}\right)=4 \pi z_{1}^{2}$. The molecular absorption loss $A_{\text {abs }}\left(f, z_{1}\right)$ can be described by the line absorption loss $A_{l a}\left(f, z_{1}\right)$ and the continuum absorption loss $A_{c a}\left(f, z_{1}\right)$ as given in [11]:

$$
A_{\mathrm{abs}}\left(f, z_{1}\right)=A_{l a}\left(f, z_{1}\right) \times A_{c a}\left(f, z_{1}\right)
$$

with $A_{l a}\left(f, z_{1}\right)$ and $A_{c a}\left(f, z_{1}\right)$ given by

$$
A_{l a}\left(f, z_{1}\right)=\exp \left(\sum_{s} k_{l a}^{s}(f) z_{1}\right)
$$

and

$$
A_{c a}\left(f, z_{1}\right)=\exp \left(\sum_{s^{\prime}} k_{c a}^{s^{\prime}}(f) z_{1}\right)
$$

where $s$ and $s^{\prime}$ are indices for molecular species and the source of the continuum absorption, respectively, and $k_{l a}$ and $k_{c a}$ are the line absorption coefficient and the continuum absorption coefficient, respectively. We utilize the am model [11], with the famous HITRAN catalog [12], to calculate the line absorption and continuum absorption coefficient.

2) Reflected path: The power spectrum $\left|H_{m, \text { refl }}\left(f, z_{m}\right)\right|^{2}$ of the reflected path is given by

$$
\left|H_{m, \text { refl }}\left(f, z_{m}\right)\right|^{2}=\left|H_{m, \operatorname{los}}\left(f, z_{m}\right) \cdot R(f)\right|^{2}
$$

where $H_{m, \operatorname{los}}\left(f, z_{m}\right)$ and $R(f)$ are the frequency response of the $m$ th path for the LoS path and the reflection coefficient, respectively. According to Kirchhoff's theory, the reflection coefficient $R(f)$ for a rough surface is given by

$$
R(f)=\gamma_{\mathrm{TE}}(f) \cdot \rho(f)
$$

where $\gamma_{\mathrm{TE}}(f)$ is the smooth surface reflection coefficient from the Fresnel equation for the transverse electric (TE) part of the electromagnetic (EM) wave and $\rho(f)$ is the Rayleigh roughness factor. Without loss of generality, this paper considers the TE part of the EM wave; the transverse magnetic (TM) part can be 

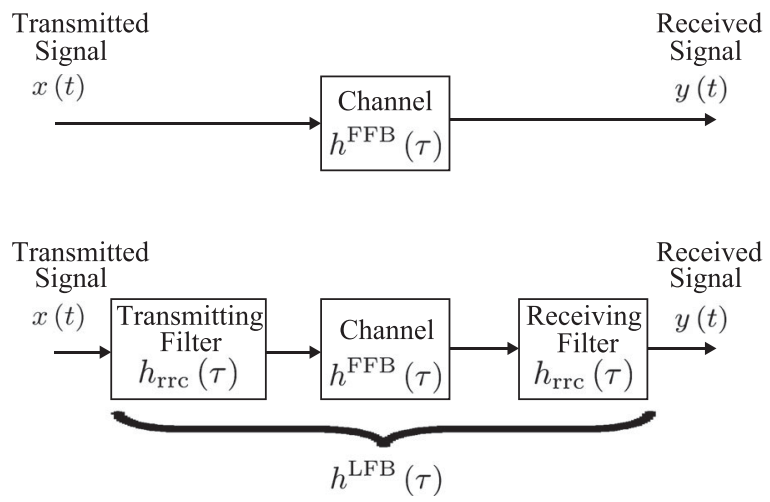

Fig. 1. Channel models for (a) the FFB and (b) the LFB scenarios.

obtained by a similar approach. The smooth surface reflection coefficient is described by

$$
\gamma_{\mathrm{TE}}(f)=\frac{n_{i} \cos \theta_{m}-n_{t} \sqrt{1-\left(\frac{n_{i}}{n_{t}} \sin \theta_{m}\right)^{2}}}{n_{i} \cos \theta_{m}+n_{t} \sqrt{1-\left(\frac{n_{i}}{n_{t}} \sin \theta_{m}\right)^{2}}}
$$

where $n_{i}, n_{t}$, and $\theta_{m}$ are the refractive index of the air, the reflector, and incident angle of $m$ th path, respectively [13]. The refractive index of the reflector $n_{t}$ is frequency dependent. But so is $n_{i}$, actually. The rough surface effect is characterized by the Rayleigh roughness factor $\rho(f)$ as

$$
\rho(f)=\exp \left(-\frac{8 \pi \cdot f^{2} \cdot \sigma^{2} \cdot \cos ^{2} \theta_{m}}{c^{2}}\right) .
$$

We assume that the height of the rough surface has a Gaussian distribution with standard deviation $\sigma$. This assumption is valid for many indoor building materials [14].

\section{B. Channel Model in the Time Domain}

Two scenarios to obtain the impulse response are considered. In the first scenario, called the full frequency band (FFB) scenario, the impulse response is characterized by the full $\mathrm{THz}$ band. In the second scenario, called the LFB scenario, the BPF is employed to focus on a certain frequency band. The block diagrams of the FFB and LFB scenarios are shown in Fig. 1.

The received signal $y(t)$ at time $t$ can be obtained by the convolution between the transmitted signal $x(t)$ and the impulse response, $h(\tau)$, as

$$
\begin{aligned}
y(t) & =\int_{-\infty}^{\infty} \sum_{m=1}^{M} h_{m}\left(\tau, z_{m}\right) x(t-\tau) d \tau \\
& =\int_{-\infty}^{\infty} h(\tau) x(t-\tau) d \tau
\end{aligned}
$$

where $h_{m}\left(\tau, z_{m}\right)$ is the impulse response of the $m$ th path. The impulse response $h(\tau)$ is described as $h^{\mathrm{FFB}}(\tau)$ in the FFB scenario and $h^{\mathrm{LFB}}(\tau)$ in the LFB scenario.

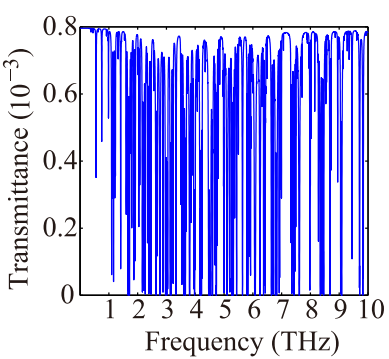

(a)

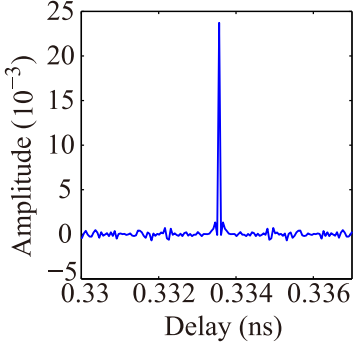

(b)
Fig. 2. (a) Transmittance $\left|H_{1, \operatorname{los}}\left(f, z_{1}\right)\right|^{2}$ as a function of frequency. (b) Impulse response $h_{\mathrm{L}}^{\mathrm{FFB}}(\tau)$ for $M=1$ for the direct path from the conventional approach as a function of delay $\tau$. Distance $z=10 \mathrm{~cm}$, pressure $p=1010 \mathrm{hPa}, \mathrm{RH}=69.6 \%$, and temperature $T=298.55 \mathrm{~K}$.

\section{Derivation of THE Phase COMPONENT}

Two criterion to obtain the phase component in the frequency response are shown in this section. The first criterion is linear phase and the second criterion is minimum phase. In the linear phase, the phase component is determined by the time shift and the frequency. On the other hand, in minimum phase, we employ Kramers-Kronig relation to determine the phase component.

\section{A. Linear Phase}

In the linear phase criterion, the phase component is set by $\phi_{\mathrm{L}, m}\left(f, z_{m}\right)=-2 \pi f z_{m} / c$. In this case, the frequency response $H_{\mathrm{L}}(f)$ is given by

$$
\begin{aligned}
H_{\mathrm{L}}(f) & =\sum_{m=1}^{M} H_{\mathrm{L}, m}\left(f, z_{m}\right) \\
& =\sum_{m=1}^{M}\left|H_{m}\left(f, z_{m}\right)\right| \exp \left(j \phi_{\mathrm{L}, m}\left(f, z_{m}\right)\right)
\end{aligned}
$$

where $\left|H_{m}\left(f, z_{m}\right)\right|$ is the square root of the power spectrum of the $m$ th path found using (4) and (7). The impulse response $h_{\mathrm{L}}^{\mathrm{FFB}}(\tau)$ is obtained by

$$
h_{\mathrm{L}}^{\mathrm{FFB}}(\tau)=\mathcal{F}^{-1}\left[H_{\mathrm{L}}(f)\right]
$$

where $\mathcal{F}^{-1}[\cdot]$ denotes the IFT operator.

If the channel has perfectly flat power spectrum with the linear phase, signal only experiences a time shift and it is perfectly causal after the time shift. In the general case, however, this is not always true. If the real and imaginary parts of the function are independent, such as in the case of real valued functions (zero phase), or linear phase imposed on real valued function, the resultant IFT is not causal (except for the special case of constant functions as discussed above). In this paper, we are concerned with $\mathrm{THz}$ frequencies $(0.1-10 \mathrm{THz})$. In this case, the received signals will experience fading due to molecular absorption and the power spectrum is, therefore, never flat (unless we look at some narrow band within this range). Therefore, the impulse response based on the linear phase cannot fulfill the causality if the channel experiences the frequency selectivity due to the molecular absorption. 
For instance, Fig. 2(a) shows the transmittance calculated with (4) as a function of frequency. Parameters used in this figure are distance $z_{1}=10 \mathrm{~cm}$, pressure $p=1010 \mathrm{hPa}, \mathrm{RH}=69.6 \%$, and temperature $T=298.55 \mathrm{~K}$. We also show the corresponding impulse response based on the linear phase shift calculated with (12)-(13) in Fig. 2(b). We can see that the main problem with the linear phase is that it causes a symmetric response around the zero (which is the propagation delay in the case of linear phase, and $\tau=0.33356 \mathrm{~ns}$ in this example). This effectively causes a response at the receiver before the arrival of the main peak. Therefore, utilization of the causal phase component should be considered in the calculation of the impulse response to ensure that the response is correct at the receiver.

\section{B. Minimum Phase}

Impulse response with causality of the $m$ th path satisfies

$$
h_{\mathrm{C}, m}^{\mathrm{FFB}}(\tau)= \begin{cases}h_{\mathrm{C}, m}^{\mathrm{FFB}}\left(\tau, z_{m}\right), & \tau \geq \tau_{m} \\ 0, & \tau<\tau_{m}\end{cases}
$$

where $\tau_{m}$ is the propagation delay of the $m$ th path and given by $\tau_{m}=z_{m} / c$. The frequency response of $h_{\mathrm{C}, m}^{\mathrm{FFB}}\left(\tau, z_{m}\right)$ is given by

$$
\begin{aligned}
H_{m}\left(f, z_{m}\right) & =\int_{\tau_{m}}^{\infty} h_{\mathrm{C}, m}^{\mathrm{FFB}}\left(\tau, z_{m}\right) e^{-j 2 \pi f \tau} d \tau \\
& =e^{-j 2 \pi f \tau_{m}} \int_{0}^{\infty} h_{\mathrm{C}, m}^{\mathrm{FFB}}\left(\tau+\tau_{m}, z_{m}\right) e^{-j 2 \pi f \tau} d \tau .
\end{aligned}
$$

$H_{m}^{\prime}\left(f, z_{m}\right)$ is defined as causal frequency response in this paper and is given by

$$
H_{m}^{\prime}\left(f, z_{m}\right)=\int_{0}^{\infty} h_{\mathrm{C}, m}^{\mathrm{FFB}}\left(\tau+\tau_{m}, z_{m}\right) e^{-j 2 \pi f \tau} d \tau
$$

which leads to

$$
H_{m}\left(f, z_{m}\right)=e^{-j 2 \pi f \tau_{m}} H_{m}^{\prime}\left(f, z_{m}\right) .
$$

Let $\exp \left[-\alpha_{m}\left(f, z_{m}\right)\right]$ and $\phi_{\mathrm{C}, m}\left(f, z_{m}\right)$ denote the amplitude and phase components of $H_{m}^{\prime}\left(f, z_{m}\right)$, respectively, i.e., $\exp \left[-\alpha_{m}\left(f, z_{m}\right)\right]=\left|H_{m}^{\prime}\left(f, z_{m}\right)\right|=\left|H_{m}\left(f, z_{m}\right)\right|$ and $\phi_{\mathrm{C}, m}\left(f, z_{m}\right)=\arg \left(H_{m}^{\prime}\left(f, z_{m}\right)\right)$. Then, $H_{m}^{\prime}\left(f, z_{m}\right)$ is given by [10], [11]

$$
H_{m}^{\prime}\left(f, z_{m}\right)=\exp \left[-\alpha_{m}\left(f, z_{m}\right)+j \phi_{\mathrm{C}, m}\left(f, z_{m}\right)\right] .
$$

$\alpha_{m}\left(f, z_{m}\right)$ and $\phi_{\mathrm{C}, m}\left(f, z_{m}\right)$ are Hilbert transform pairs from the Kramers-Kronig relation [10], [11]. Therefore, the impulse response $h_{\mathrm{C}, m}^{\mathrm{FFB}}\left(\tau, z_{m}\right)$ satisfies causality. Hence, $\phi_{\mathrm{C}, m}\left(f, z_{m}\right)$ is given by

$$
\phi_{\mathrm{C}, m}\left(f, z_{m}\right)=\frac{1}{\pi} \mathrm{PV} \int_{-\infty}^{\infty} \frac{\alpha_{m}\left(f^{\prime}, z_{m}\right)}{f-f^{\prime}} d f^{\prime}
$$

where PV represents Cauchy principal value [15]. The criterion to set the phase component in (19) is defined as minimum phase in this paper. Given $\left|H_{m}\left(f, z_{m}\right)\right|, H_{m}\left(f, z_{m}\right)$ is available based on (17)-(19). Finally, the impulse response with the minimum phase of the multipath is given by

$$
h_{\mathrm{C}}^{\mathrm{FFB}}(\tau)=\mathcal{F}^{-1}\left[\sum_{m=1}^{M} H_{m}\left(f, z_{m}\right)\right] .
$$

From the above relation, the impulse response with minimum phase is calculated as follows. First, $\alpha_{m}\left(f, z_{m}\right)$ is obtained from (4) or (7) and then substituted in (19). Finally, (20) is obtained from (17), calculated using (18).

In the LFB scenario, the impulse response with the minimum phase $h_{\mathrm{C}}^{\mathrm{LFB}}(\tau)$ can be obtained by

$$
h_{\mathrm{C}}^{\mathrm{LFB}}\left(\tau, f_{c}\right)=\int_{-\infty}^{\infty} h_{\mathrm{C}}^{\mathrm{FFB}}\left(\tau^{\prime}\right) h_{r c}\left(\tau-\tau^{\prime}, f_{c}\right) d \tau^{\prime}
$$

where $h_{r c}\left(\tau, f_{c}\right)$ represents the effect of the BPFs. We employ root raised cosine filters $h_{\mathrm{rrc}}\left(\tau, f_{c}\right)$ as the BPF at both transmitter and receiver. Let $h^{\mathrm{LFB}}$ indicate the impulse response involving $h^{\mathrm{FFB}}$ and the BPFs. The effect of the BPFs $h_{r c}\left(\tau, f_{c}\right)$ is given by [16]

$$
\begin{aligned}
h_{r c}\left(\tau, f_{c}\right)= & 2 \pi^{2} f_{o} \operatorname{sinc}\left(2 \pi f_{o} \tau\right) \frac{\cos \left(2 a \pi f_{o} \tau\right)}{\pi^{2}-\left(4 a \pi f_{o} \tau\right)^{2}} \\
& \times \cos \left(2 \pi f_{c} \tau\right)
\end{aligned}
$$

where $f_{o}=(\pi B) /(2 \pi+4.853 a), a$ is the roll-off coefficient of $\mathrm{BPF}, B$ is the transmission band, and $f_{c}$ is the center frequency. In the LFB scenario, $f_{c}$ and $B$ determine the target frequency band. In practice, the filter response must be delayed and truncated to satisfy causality [17]-[19]. Therefore, we truncate the extreme ends of the above filter response and delay the response such that the first time-domain component is at time zero after the truncation.

\section{NUMERICAL AND EXPERIMENTAL RESULTS}

In this section, we will show the validity of the impulse response with minimum phase, the coherence bandwidth, and the power loss for the LFB scenario.

The common parameters used in all the numerical evaluations are as follows: pressure $p=1010 \mathrm{hPa}, \mathrm{RH}=69.6 \%$, and temperature $T=298.55 \mathrm{~K}$, unless otherwise indicated. The roll-off coefficient $a=1$ for $h_{\mathrm{rc}}$.

\section{A. Comparison Between Measurement and Analysis}

To validate the impulse response with minimum phase, a comparison of the received pulse based on measurements and the received pulse based on analysis is performed. The measurements were made at distance $z_{1}=62.5 \mathrm{~cm}$, temperature $T=295.15 \mathrm{~K}$, and pressure $p=1015.9 \mathrm{hPa}$. Measurements were conducted by terahertz time domain spectroscopy $(\mathrm{THz}-$ TDS) technique using the TeraVil-EKSPLA T-spec spectrometer. The experimental measurement setup is shown in Fig. 3. The measurement setup consists of the pump source, the laser delay lines, the medium control, and the signal measurement block. The delay lines are for obtaining $\mathrm{THz}$ pulse shape in time domain. The medium control is for setting channel medium. In order to measure $\mathrm{THz}$ signal in a LoS there is no sample at the sample location. The emitter focus on the detector in THz optics 


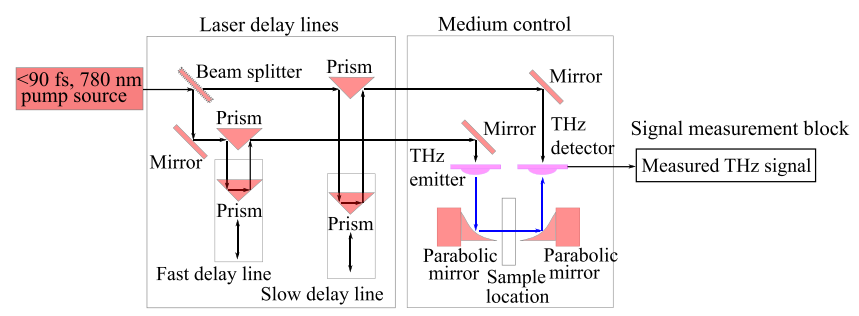

Fig. 3. Terahertz time domain spectroscopy setup.

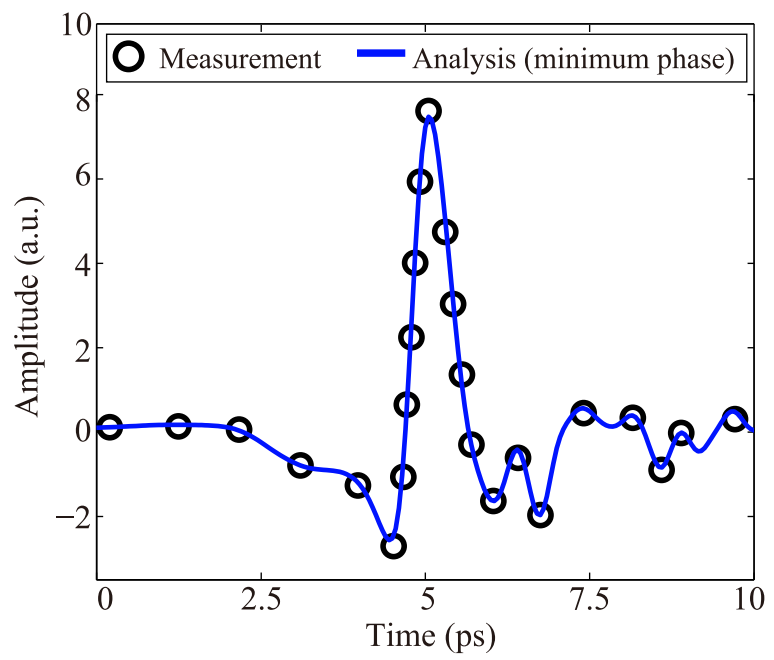

Fig. 4. Received pulse comparison between the measurement result at $\mathrm{RH}=$ $52 \%$ and analytically obtained result based on the minimum phase in the timedomain.

in Fig. 3 with a beam width of approximately $2 \mathrm{~cm}$ at the sample location.

There were two measurements, one at $\mathrm{RH}=52 \%$ and the other at $\mathrm{RH}=6 \%$. The received pulse at $\mathrm{RH}=6 \%$ is used as the transmitted signal $x(t)$, since molecular absorption is small. The received pulse is obtained analytically by the transmitted signal and either by the impulse responses with minimum phase $\left(h_{\mathrm{C}}^{\mathrm{FFB}}\right)$ or the impulse response with linear phase $\left(h_{\mathrm{L}}^{\mathrm{FFB}}\right)$ [8].

Comparison between the received pulses at $\mathrm{RH}=52 \%$ is shown in Figs. 4 and 5. The analytically obtained pulse from $h_{\mathrm{C}}^{\mathrm{FFB}}$ agrees well with the received pulse from the measurement data and it satisfies causality, as shown in Fig. 4. The analytically obtained received pulse from $h_{\mathrm{L}}^{\mathrm{FFB}}$ implies that causality is not satisfied as shown in Fig. 5. Although the difference between the analytically obtained received pulse from $h_{\mathrm{L}}^{\mathrm{FFB}}$ and the measurement result is relatively large, it is not very significant. This is because the frequency response does not have much frequency selectivity. This can be seen in Fig. 6, where the power spectrum at $\mathrm{RH}=6 \%$ and $\mathrm{RH}=52 \%$ correspond to the transmitted power spectrum and received power spectrum, respectively.

For comparison in a frequency band with significant frequency selectivity, we must carry out the measurement in a higher frequency band, such $1.5 \mathrm{THz}$, which is difficult due to limitations of the measurement equipment. Alternatively, we compare the analytically received pulses provided by $h_{\mathrm{C}}^{\mathrm{FFB}}$ and

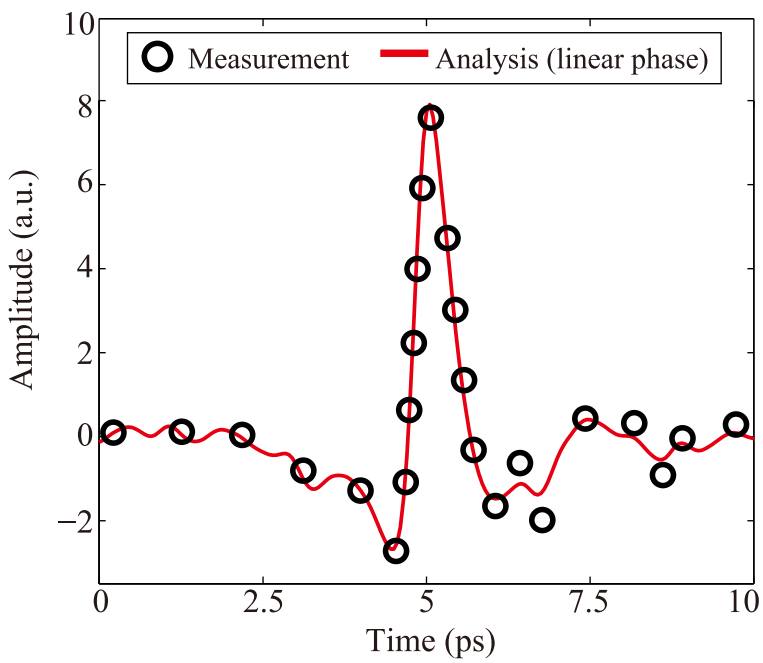

Fig. 5. Received pulse comparison between the measurement result at $\mathrm{RH}=52 \%$ and analytically obtained result based on the linear phase in the time-domain

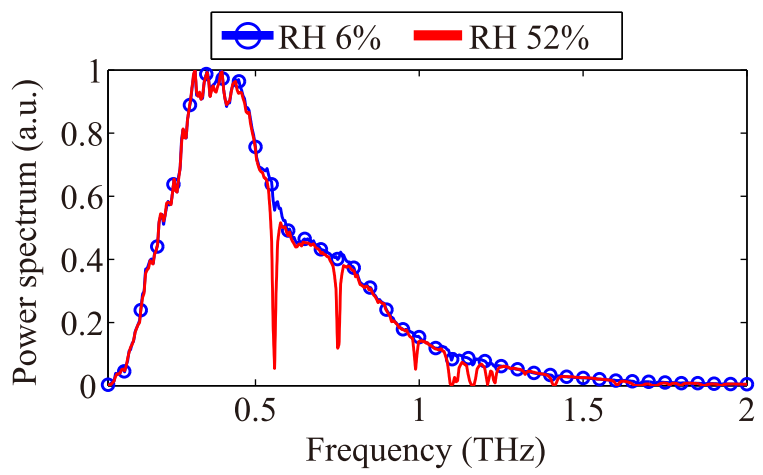

Fig. 6. Comparison between transmitted power spectrum at $\mathrm{RH}=6 \%$ and received power spectrum at $\mathrm{RH}=52 \%$ with bandwidth $0.9 \mathrm{THz}$ at $0.4 \mathrm{THz}$. Distance $z_{1}=62.5 \mathrm{~cm}$, temperature $T=295.15 \mathrm{~K}$, and pressure $p=1015.9 \mathrm{hPa}$.

$h_{\mathrm{L}}^{\mathrm{FFB}}$ in Fig. 7. Here, the power spectrum has the most power from $1 \mathrm{THz}$ to $2 \mathrm{THz}$, and we can see frequency selectivity in Fig. 7(b). Fig. 7(a) shows the analytically obtained received pulses based on $h_{\mathrm{C}}^{\mathrm{FFB}}$ and $h_{\mathrm{L}}^{\mathrm{FFB}}$. Due to frequency selectivity, the effect of the delay wave is significant in $h_{\mathrm{C}}^{\mathrm{FFB}}$. In addition, the difference between $h_{\mathrm{C}}^{\mathrm{FFB}}$ and $h_{\mathrm{L}}^{\mathrm{FFB}}$ is now significant, indicating the validity and importance of $h_{\mathrm{C}}^{\mathrm{FFB}}$ for the practical THz pulse shape. In addition, the result indicates that only $h_{\mathrm{C}}^{\mathrm{FFB}}$ satisfies causality.

We also evaluate the importance of the minimum phase in energy detection based wireless communication scenario. Specifically, the effect of leak power due to delayed component in the received signal is evaluated in the case of Fig. 7. Gaussian pulse for transmitted signal and On-Off keying modulation are assumed. The transmitted signal in case of one pulse transmission is plotted in Fig. 8. The received signals of the impulse response with linear phase and the impulse response with minimum phase are plotted in Figs. 9 and 10, respectively. At the receiver, the energy detector is employed and the time duration for detecting the pulse is set to $T=1.025 \mathrm{ps}$ according to the 


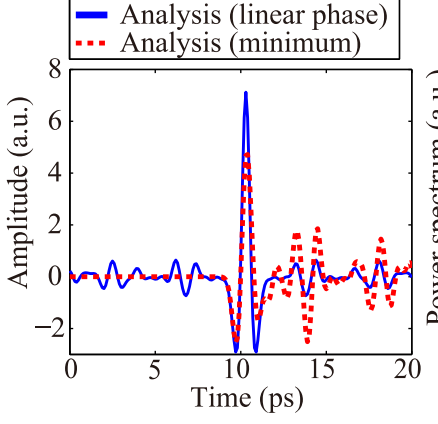

(a)

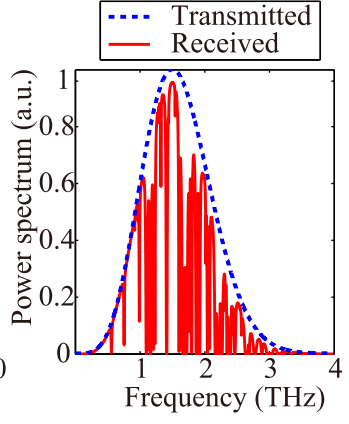

(b)
Fig. 7. Comparison between the linear phase result and minimum phase result with bandwidth $2.2 \mathrm{THz}$ at $1.5 \mathrm{THz}$ at $\mathrm{RH}=52 \%$. (a) Received pulse in time domain (b) Transmitted power spectrum, $\mathrm{RH}=6 \%$ and received power spectrum, $\mathrm{RH}=52 \%$. Distance $z_{1}=62.5 \mathrm{~cm}$, temperature $T=295.15 \mathrm{~K}$, and pressure $p=1015.9 \mathrm{hPa}$.

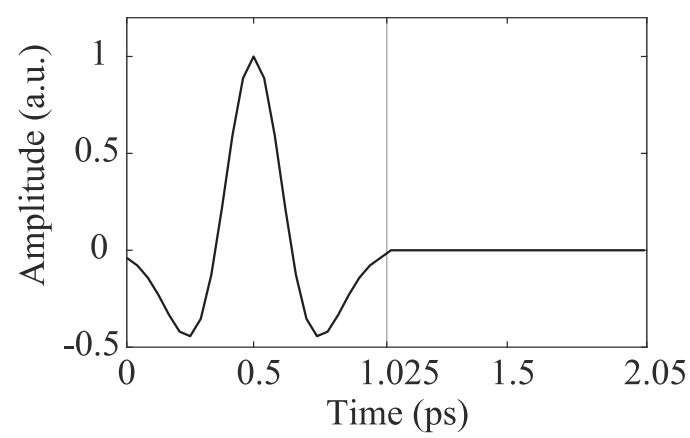

Fig. 8. Transmitted Gaussian pulse with bandwidth $2.2 \mathrm{THz}$ at $1.5 \mathrm{THz}$.

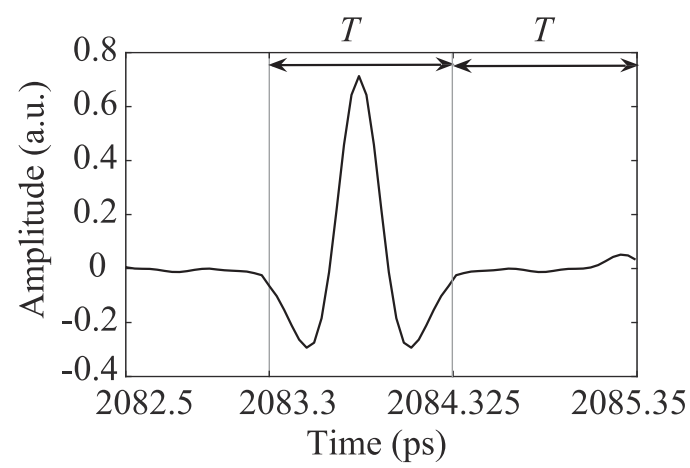

Fig. 9. Received pulse obtained from linear phase at $\mathrm{RH}=52 \%$ with bandwidth $2.2 \mathrm{THz}$ at $1.5 \mathrm{THz}$. Distance $z_{1}=62.5 \mathrm{~cm}$, temperature $T=295.15 \mathrm{~K}$, and pressure $p=1015.9 \mathrm{hPa}$.

transmitted signal in Fig. 8. Since the arrival time $\tau_{m}=2083.3$ ps, the energy detection output $E_{\text {main }}$ is given by

$$
E_{\text {main }}=\int_{\tau_{m}}^{\tau_{m}+T}|y(t)|^{2} d t .
$$

We define $E_{\text {main }}$ as main power which contributes for the energy detection. We also define the leak power as

$$
E_{\text {leak }}=\int_{\tau_{m}+T}^{\tau_{m}+2 T}|y(t)|^{2} d t
$$

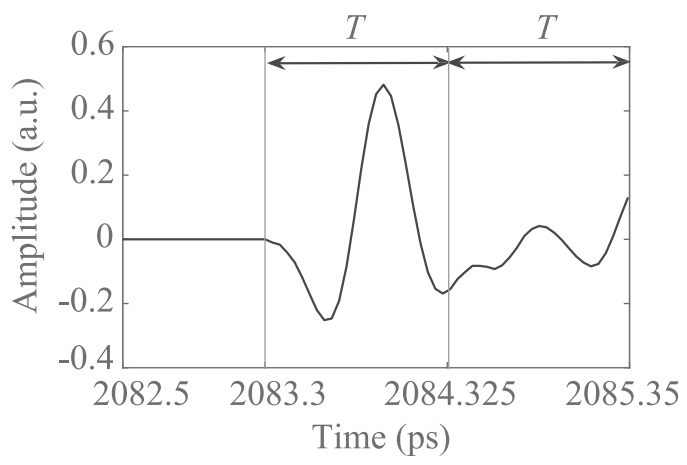

Fig. 10. Received pulse obtained from minimum phase at $\mathrm{RH}=52 \%$ with bandwidth $2.2 \mathrm{THz}$ at $1.5 \mathrm{THz}$. Distance $z_{1}=62.5 \mathrm{~cm}$, temperature $T=$ $295.15 \mathrm{~K}$, and pressure $p=1015.9 \mathrm{hPa}$.

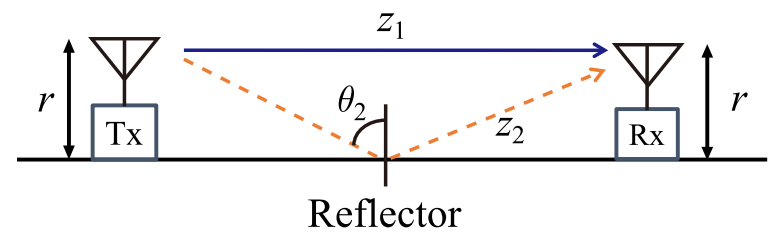

Fig. 11. Multipath scenario $(M=2)$ with LoS and reflected path.

This leak power corresponds to intersymbol interference power. In order to evaluate the significance of the impulse response, we use main power to leak power ratio (MLR) since MLR has to be used to design the symbol time duration $(T)$, and countermeasures to the intersymbol interference, such as error correcting coding, equalization. In this case, MLR for linear phase and minimum phase are $23.78 \mathrm{~dB}$ and $9.61 \mathrm{~dB}$, respectively. This result indicates that the significance of the minimum phase for the impulse response derivation.

\section{B. Impulse Response for Multipath}

We assume a two path model, where one is a LoS path (solid arrow) and the other one is a reflected path (dashed arrow) as shown in Fig. 11. In this scenario, the height of both transmitter antenna and receiver antenna, the distance of the LoS path, and the distance of the second path are $r, z_{1}$, and $z_{2}$, respectively. $\theta_{m}$ is the angle of the incident wave to the reflector. We assume that the reflector is made of plaster, which is usually used in walls and its refractive index $n_{t}$ is 2.24 . The reflector has the rough surface height standard deviation $\sigma=0.0088 \mathrm{~cm}$. In our multipath simulation, the parameters are as follows: $n_{i}=1$, $r=1 \mathrm{~cm}$, and $z_{1}=1-100 \mathrm{~cm}$.

Figs. 12 and 13 show impulse responses for multipath environments for two distances: $z_{1}=10 \mathrm{~cm}$ and $z_{1}=80 \mathrm{~cm}$, respectively. In these figures, relative time $=0$ corresponds to the time when the first wave arrives and the $6.6 \mathrm{ps}$ and $0.8 \mathrm{ps}$ markers are the relative arrival times of the reflected wave, which is given by $\tau_{2}-\tau_{1}$, in Figs. 12 and 13 (dashed lines). The difference between the LoS path distance $z_{1}$ and the reflected path distance $z_{2}$ decreases when $z_{1}$ increases. At the same time, spreading loss and molecular absorption loss increase as the LoS path distance $z_{1}$ increases. The reflected path also suffers 


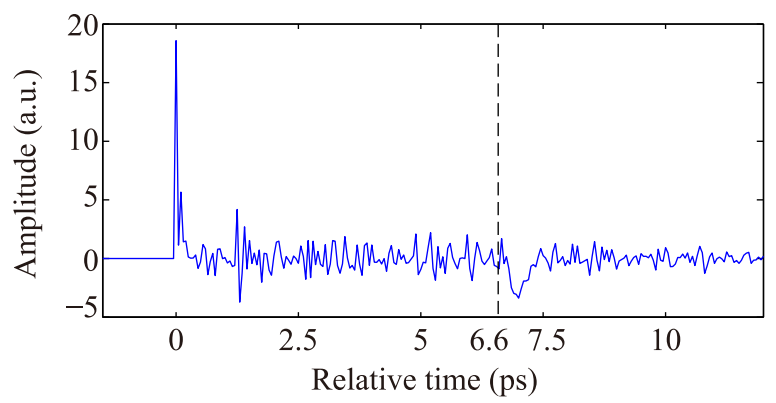

Fig. 12. Impulse response for multipath environment in $z_{1}=10 \mathrm{~cm}$.

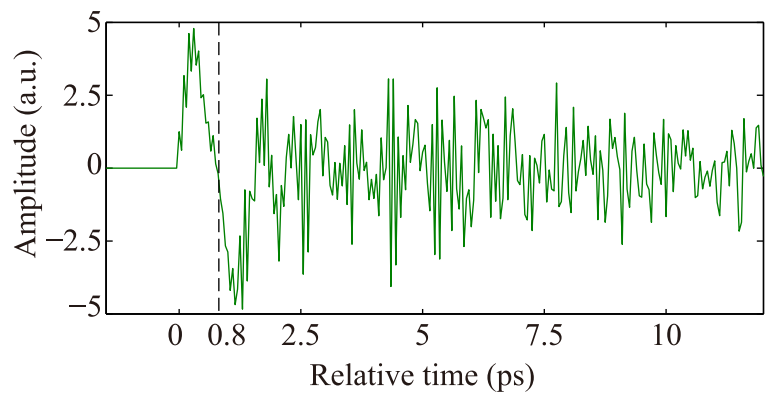

Fig. 13. Impulse response for multipath environment in $z_{1}=80 \mathrm{~cm}$.

from spreading loss and molecular absorption. Therefore, the absolute energy of the reflected path is smaller for $80 \mathrm{~cm}$. However, the relative impact may be smaller for the $10 \mathrm{~cm}$ case compared to the $80 \mathrm{~cm}$ case.

\section{Coherence Bandwidth}

In this section, we show coherence bandwidth for the FFB and LFB scenarios. The coherence bandwidth $W^{\mathrm{Coh}}$ of a causal channel is, in general, given by the inverse of the root mean square delay spread $\tau_{\text {rms }}$ [16], [20]

$$
W^{\mathrm{Coh}}=1 / \tau_{\text {rms }} \text {. }
$$

The root mean square delay spread $\tau_{\text {rms }}$ is defined by [16], [20]

$$
\tau_{\text {rms }}=\sqrt{\frac{\int(\tau-\bar{\tau})^{2}|h(\tau)|^{2} d \tau}{\int|h(\tau)|^{2} d \tau}}
$$

where $h(\tau)$ is the impulse response and $\bar{\tau}$ is mean delay time defined by [16], [20]

$$
\bar{\tau}=\frac{\int \tau|h(\tau)|^{2} d \tau}{\int|h(\tau)|^{2} d \tau} .
$$

In the UHF band, multipath channel is caused by obstacles between a transmitter and a receiver. Specifically, the transmitted signal is reflected by the obstacles in the propagation environment. Thus, the reflected signals reach the receiver through different paths. Each path has a different delay that causes a different phase shift. The difference in the phase shifts results either in constructive or destructive fading in the frequency response. Therefore, the coherence bandwidth, which represents an approximately flat frequency bandwidth, is determined by the delay time of each path. Since the delay time is random, the

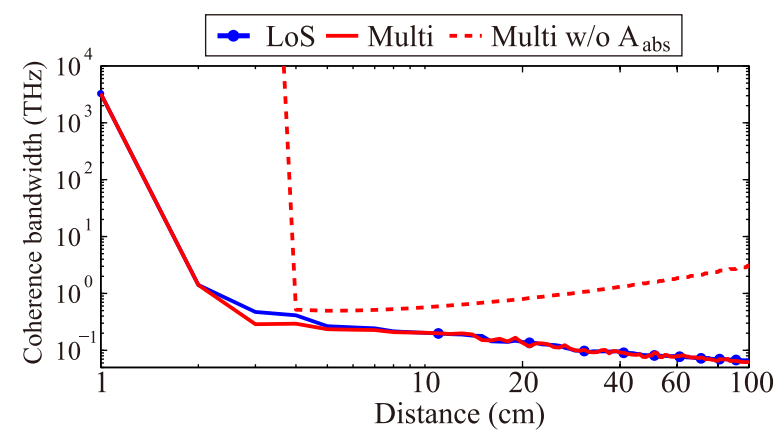

Fig. 14. Coherence bandwidth for impulse response with the minimum phase for a distance $z_{1}$ ranging from 1 to $100 \mathrm{~cm}$.

coherence bandwidth in the LFB scenario in the UHF band is also random [21].

The THz band multipath channel affected by not only reflected signals but also molecular absorption. Reflected signals with random delays result in random fading in the frequency domain. In addition, molecular absorption depends on the pressure, temperature, $\mathrm{RH}$, and the distance between the transmitter and receiver. Fading due to molecular absorption is approximately deterministic because of the vast numbers of molecules in the air. THz band channels have both random and deterministic fading characteristics. Thus, the behavior of the coherence bandwidth is complex. Since the coherence bandwidth corresponds to average flat bandwidth, this information is useful when designing short-range $\mathrm{THz}$ band wireless communication systems.

1) Coherence Bandwidth for FFB: The coherence bandwidth $W_{\mathrm{FFB}, \text { los }}^{\mathrm{Coh}}\left(z_{1}\right)$ of the LoS path is plotted with a solid line with circles and the coherence bandwidth $W_{\mathrm{FFB}, \text { multi }}^{\mathrm{Coh}}\left(z_{1}\right)$ of the multipath is plotted as a solid line in Fig. 14. The dashed line represents the coherence bandwidth for the multipath without molecular absorption in Fig. 14. The multipath without molecular absorption implies $A_{\mathrm{abs}}\left(f, z_{m}\right)=1$ in (4). In the calculation of the coherence bandwidth, the components that are less than $-30 \mathrm{~dB}$ from the peak value are not considered. A similar approach was adopted in [22].

The coherence bandwidth for the multipath without molecular absorption is wider than $W_{\mathrm{FFB}, \text { los }}^{\mathrm{Coh}}\left(z_{1}\right)$ and $W_{\mathrm{FFB}, \text { multi }}^{\mathrm{Coh}}\left(z_{1}\right)$. Furthermore, $W_{\mathrm{FFB}, \text { los }}^{\mathrm{Coh}}\left(z_{1}\right)$ and $W_{\mathrm{FFB}, \text { multi }}^{\mathrm{Coh}}\left(z_{1}\right)$ show a similar behavior. Thus, we find that the impact of molecular absorption is dominant on the coherence bandwidth in the FFB scenario. $W_{\mathrm{FFB}, \text { los }}^{\mathrm{Coh}}$ and $W_{\mathrm{FFB}, \mathrm{multi}}^{\mathrm{Coh}}$ decrease as the distance $z_{1}$ increases. In the range of $1-20 \mathrm{~cm}, W_{\mathrm{FFB}, \text { los }}^{\mathrm{Coh}}$ and $W_{\mathrm{FFB} \text {,multi }}^{\mathrm{Coh}}$ decrease rapidly, but above $40 \mathrm{~cm}$ the coherence bandwidths converge.

To give a reason for this behavior, we plotted transmittances for the LoS paths at distances $z_{1}=2 \mathrm{~cm}, 10 \mathrm{~cm}$, and $80 \mathrm{~cm}$ in Fig. 15. It can be observed that the molecular absorption loss increases significantly with distance according to the Beer-Lambert law, resulting in a narrower $W_{\mathrm{FFB}}^{\mathrm{Coh}}$ as $z_{1}$ increases. The dominant molecular absorption loss at the higher distances causes the above coherence bandwidths to converge. In addition, the flat bandwidth is observed to be dependent on frequency. 


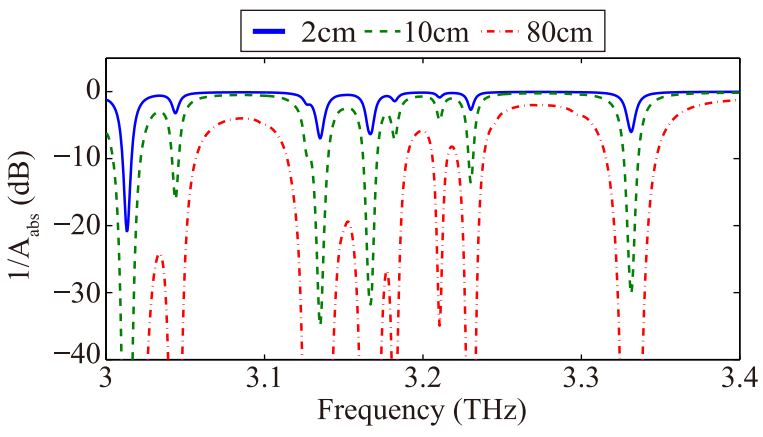

Fig. 15. Comparison of $1 / A_{\text {abs }}$ for distance $z_{1}=2,10$, and $80 \mathrm{~cm}$ for the frequency range $3-3.4 \mathrm{THz}$.
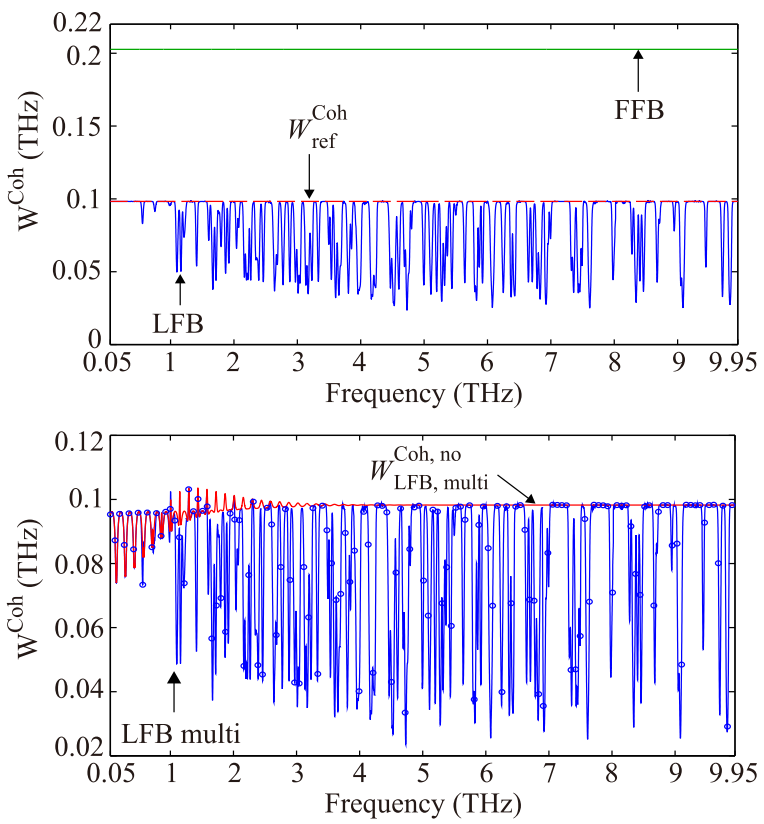

Fig. 16. Comparison between coherence bandwidths for $B=0.05 \mathrm{THz}$ at $10 \mathrm{~cm}$.

2) Coherence Bandwidth for LFB: We evaluate the achievable coherence bandwidth $W_{\mathrm{LFB}, \mathrm{Cos}}^{\mathrm{Coh}}$ in the LFB scenario for the LoS path and $W_{\mathrm{LFB} \text {, multi }}^{\mathrm{Coh}}$ for the multipath channel in Figs. 1619. $W_{\mathrm{LFB}, \text { los }}^{\mathrm{Coh}}$ and $W_{\mathrm{LFB}, \mathrm{Coh}}^{\mathrm{Colti}}$ are obtained from $h_{\mathrm{C}, 1}^{\mathrm{LFB}}$ and $h_{\mathrm{C}}^{\mathrm{LFB}}$, respectively, for a given center frequency $f_{c}$ and the required transmission band $B$.

In Figs. 16-19, $W_{\mathrm{LFB}, \text { los }}^{\mathrm{Coh}}$ and $W_{\mathrm{LFB}, \text { multi }}^{\mathrm{Coh}}$ as functions of the center frequency $f_{c}$ are plotted with different BPFs, i.e., $B=0.05$ and $0.3 \mathrm{THz}$, respectively. In this case, coherence bandwidths $W_{\text {ref }}^{\text {Coh }}(B)$ of the BPF (without channel, only with BPFs) are $0.0983 \mathrm{THz}$ and $0.5895 \mathrm{THz}$, respectively. These are plotted in dashed lines in these figures. The distance $z_{1}$ is set to $10 \mathrm{~cm}$ in Figs. 16 and 17 and to $80 \mathrm{~cm}$ in Figs. 18 and 19. In these figures, the coherence bandwidths for FFB $W_{\mathrm{FFB}, \text { los }}^{\mathrm{Coh}}$ with $10 \mathrm{~cm}$ and $80 \mathrm{~cm}$ are also plotted.

First, we show the common features of the LoS path and multipath. We can see that $W_{\mathrm{LFB}, \mathrm{Cos}}^{\mathrm{Coh}}$ and $W_{\mathrm{LFB}, \mathrm{Coh}}^{\mathrm{Colti}}$ change significantly when $f_{c}$ changes. This is due to the frequency selective molecular absorption loss and multipath fading.
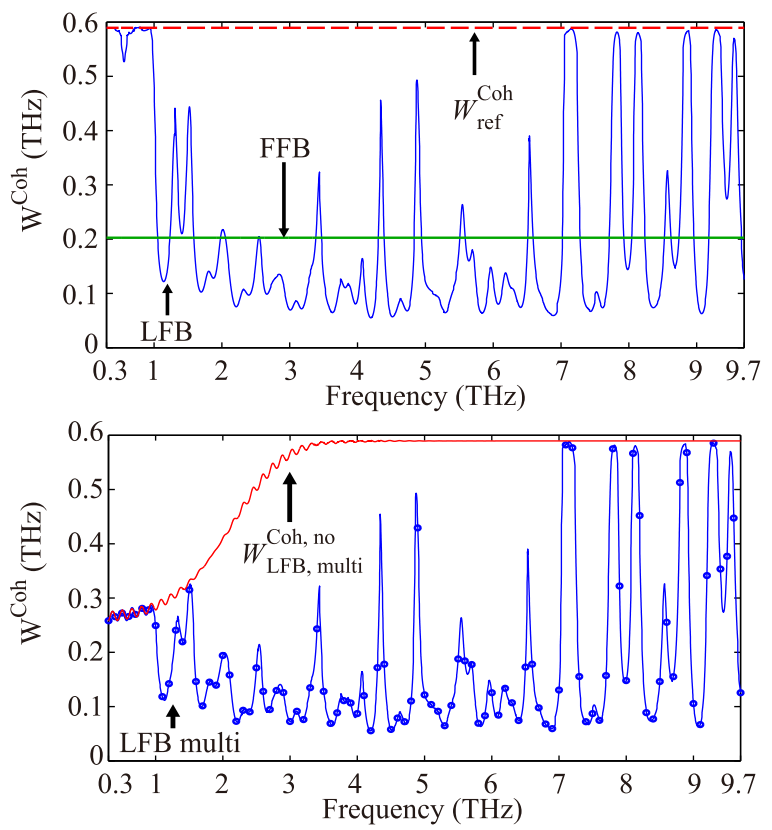

Fig. 17. Comparison between coherence bandwidths for $B=0.3 \mathrm{THz}$ at $10 \mathrm{~cm}$.
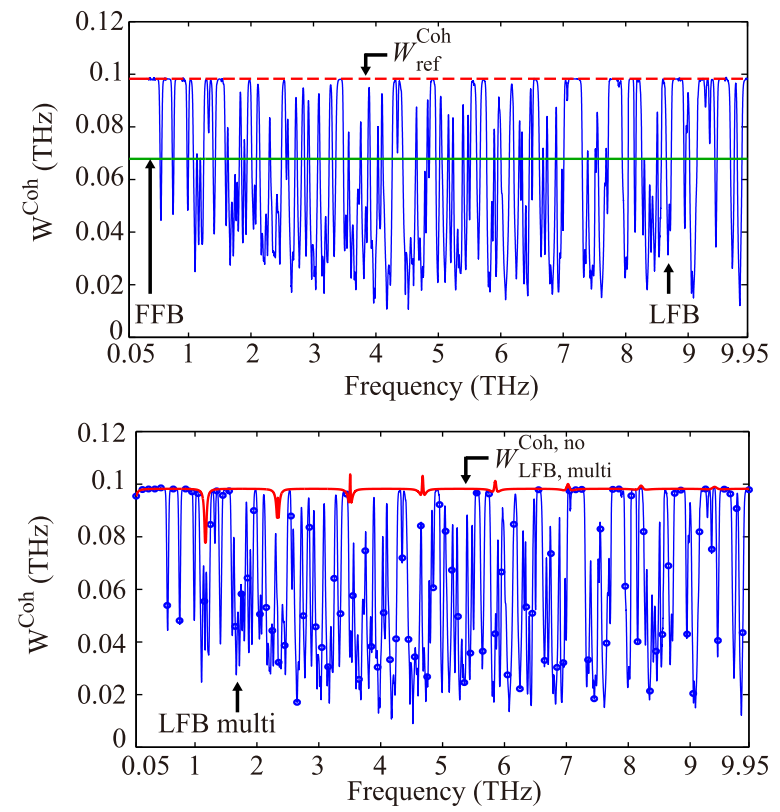

Fig. 18. Comparison between coherence bandwidths for $B=0.05 \mathrm{THz}$ at $80 \mathrm{~cm}$.

Increasing the bandwidth and distance leads to fewer frequencies that can be close to $W_{\mathrm{ref}}^{\mathrm{Coh}}(B)$. The upper bound of $W_{\mathrm{LFB}, \mathrm{Cos}}^{\mathrm{Coh}}$ is approximately equal to $W_{\mathrm{ref}}^{\mathrm{Coh}}(B)$ since $B$ limits the coherence bandwidth of $W_{\mathrm{LFB}}^{\mathrm{Coh}} . W_{\mathrm{ref}}^{\mathrm{Coh}}(B)$ is limited by the required transmission band and is therefore independent of $W_{\mathrm{FFB}, \text { los }}^{\mathrm{Coh}}$.

Second, we focus on the frequency band where multipath fading is dominant. Below $1 \mathrm{THz}$ in Figs. 16 and 17, multipath is the most significant effect. In this frequency band, there is only a little effect from molecular absorption [see Fig. 2(a)]. Accordingly, the coherence bandwidth $W_{\mathrm{LFB}, \mathrm{Coh}}^{\mathrm{Coh}}$ is 

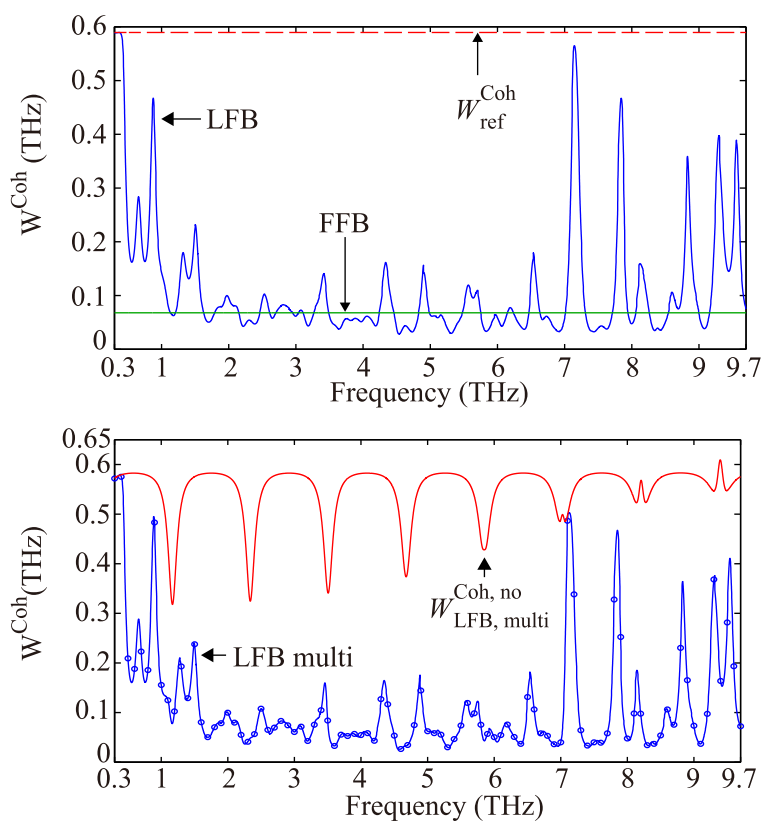

Fig. 19. Comparison between coherence bandwidths for $B=0.3 \mathrm{THz}$ at $80 \mathrm{~cm}$.

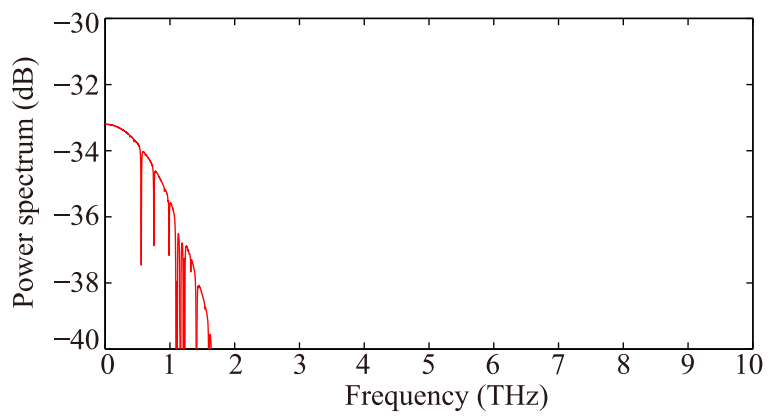

Fig. 20. Power spectrum of reflected wave without spreading loss for $z_{1}=$ $10 \mathrm{~cm}$.

approximately equal to $W_{\mathrm{LFB}, \mathrm{Coh} \text {,no }}^{\mathrm{Co}}$. $W_{\mathrm{LFB}, \mathrm{multi}}^{\mathrm{Coh}, \mathrm{no}}$ is the coherence bandwidth without molecular absorption in multipath. As mentioned above, the multipath model without molecular absorption means $A_{\mathrm{abs}}=1$. Fig. 20 shows the power spectrum of the reflected wave at $10 \mathrm{~cm}$. The peak power of the reflected wave lies below $1 \mathrm{THz}$, where also the multipath effect is the strongest.

Finally, we show the results for the frequency band where molecular absorption is dominant. Figs. 18 and 19 show that molecular absorption is dominant in frequencies above $1 \mathrm{THz}$. Fig. 21 shows the power spectrum of the reflected wave at $80 \mathrm{~cm}$. From this figure, we can see that the reflected paths affect the entire $\mathrm{THz}$ band. However, a distance between notches of $W_{\mathrm{LFB}, \text { multi }}^{\mathrm{Coh} \text {,no }}$ is wider than $W_{\mathrm{LFB}, \text { multi }}^{\mathrm{Coh}}$ in Figs. 18 and 19; therefore, $W_{\mathrm{LFB}, \mathrm{multi}}^{\mathrm{Coh}}$ is very similar to $W_{\mathrm{LFB}, \mathrm{los}}^{\mathrm{Coh}}$ at $80 \mathrm{~cm}$.

\section{Channel Power Loss}

We investigate channel power loss in this section. Here, we assume the LFB scenario and an impulse-based transmitted signal.

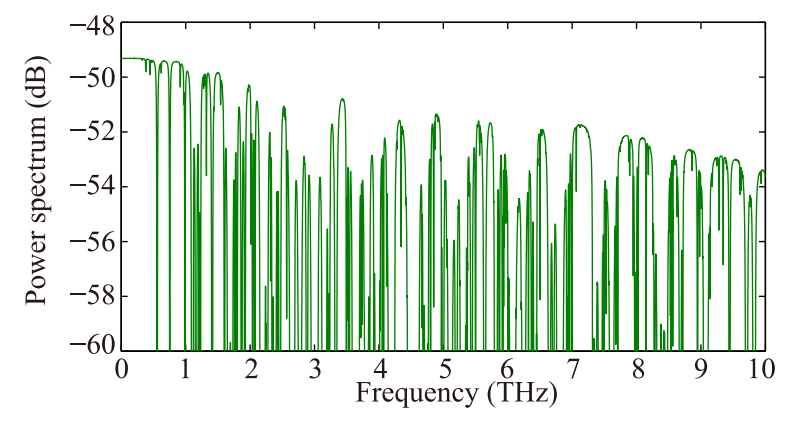

Fig. 21. Power spectrum of reflected wave without spreading loss for $z_{1}=$ $80 \mathrm{~cm}$.

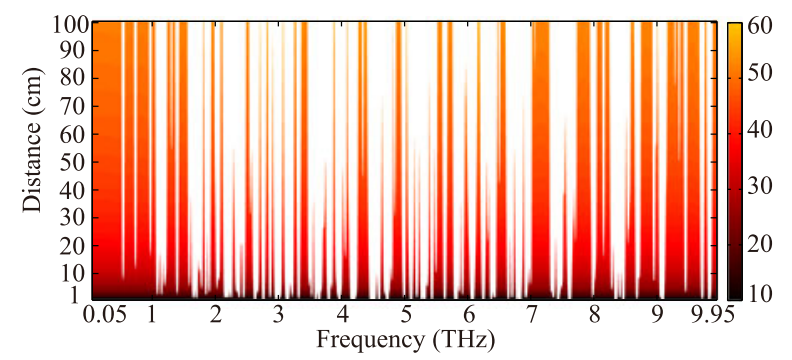

Fig. 22. Channel power loss for $B=0.05 \mathrm{THz}$ in the LoS path for a distance range $1-100 \mathrm{~cm}$.

We define the channel power loss $\Phi\left(z_{1}, f_{c}, B\right)$ as

$$
\begin{aligned}
\Phi & \left(z_{1}, f_{c}, B\right) \\
= & \begin{cases}P_{\text {loss }}\left(z_{1}, f_{c}, B\right), & \left(\begin{array}{l}
W_{\mathrm{LFB}}^{\mathrm{Coh}}\left(z_{1}, f_{c}, B\right) \geq W_{\text {target }}^{\mathrm{Coh}}(B) \\
\& P_{\text {loss }}\left(z_{1}, f_{c}, B\right) \leq P_{\text {loss }}^{\text {target }}
\end{array}\right) \\
0, & \text { (otherwise) }\end{cases}
\end{aligned}
$$

where $W_{\text {target }}^{\text {Coh }}(B)$ denotes a target coherence bandwidth and, without loss of generality, it is assumed to be $0.9 W_{\mathrm{ref}}^{\mathrm{Coh}}(B)$. $P_{\text {loss }}$ is the energy loss due to spreading, molecular absorption loss, and multipath fading. $P_{\text {loss }}^{\text {target }}$ is the target power loss and, it is assumed to be $60 \mathrm{~dB}$. $\Phi\left(z_{1}, f_{c}, B\right)$ represents the power loss with a given distance $z_{1}$ and frequency band $\left(f_{c}\right.$ and $\left.B\right)$. In addition, $\Phi\left(z_{1}, f_{c}, B\right)=0$ indicates that the coherence bandwidth does not satisfy $W_{\text {target }}^{\text {Coh }}(B)$ and/or that the power loss exceeds $P_{\text {loss }}^{\text {target }}$. On the other hand, $\Phi\left(z_{1}, f_{c}, B\right)>0$ indicates that both $W_{\text {target }}^{\text {Coh }}(B)$ and $P_{\text {loss }}^{\text {target }}$ are satisfied.

Results for the channel power loss for the LoS path with $B=$ 0.05 and $0.3 \mathrm{THz}$ are shown in Figs. 22 and 23, respectively. Target coherence bandwidths for $B=0.05$ and $0.3 \mathrm{THz}$ are $0.0884 \mathrm{THz}$ and $0.5306 \mathrm{THz}$, respectively. In Figs. 22 and 23, the colored area represents the power loss $P_{\text {loss }}($ in $\mathrm{dB}$ ) as a function of distance and center frequency and the white areas in the figure represent $\Phi\left(z_{1}, f_{c}, B\right)=0$.

We can see that $P_{\text {loss }}$ mostly depends on the center frequency. The feasible frequency bands, achieving $\Phi\left(z_{1}, f_{c}, B\right) \neq 0$, significantly decrease as $B$ increases, as shown in Figs. 22 and 23. In addition, the feasible frequency bands decrease gradually when the distance $z_{1}$ is increased. This behavior is caused by molecular absorption loss as shown in Section IV-C2. 


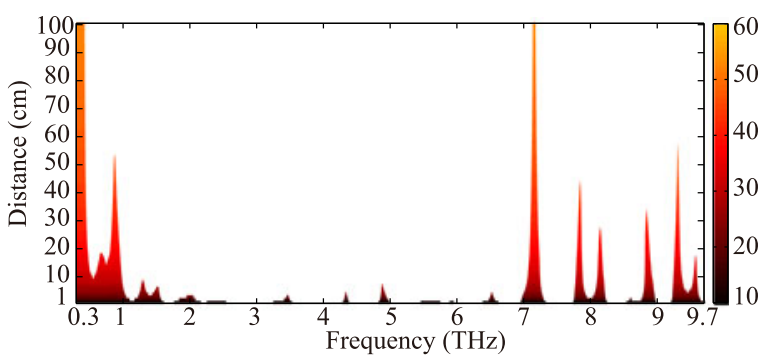

Fig. 23. Channel power loss for $B=0.3 \mathrm{THz}$ in the LoS path for a distance range $1-100 \mathrm{~cm}$.

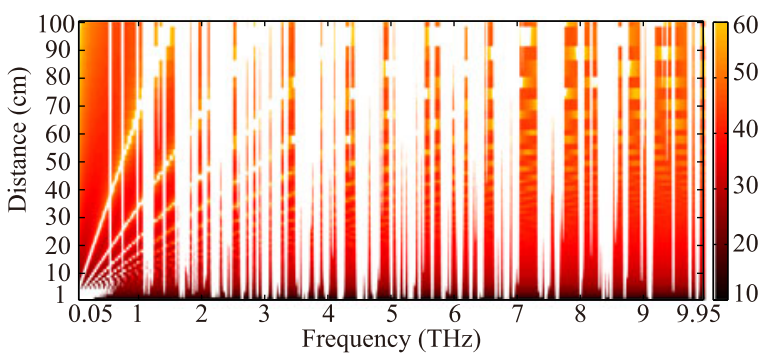

Fig. 24. Channel power loss for $B=0.05 \mathrm{THz}$ in the multipath for a distance range $1-100 \mathrm{~cm}$.

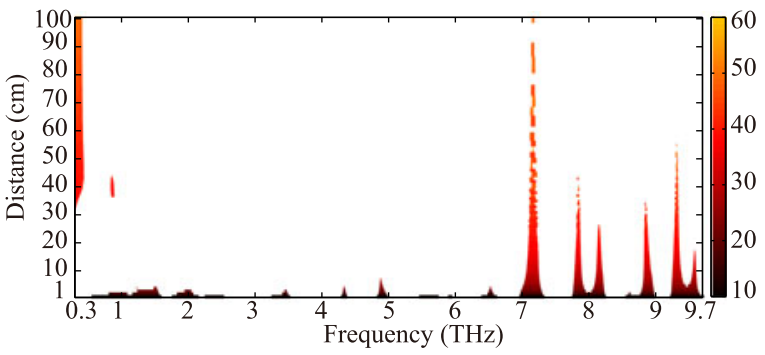

Fig. 25. Channel power loss for $B=0.3 \mathrm{THz}$ in the multipath for a distance range $1-100 \mathrm{~cm}$.

Results for the channel power loss in the multipath channel with $B=0.05$ and $0.3 \mathrm{THz}$ are shown in Figs. 24 and 25, respectively. Target coherence bandwidths are the same as in the case of LoS path. Colored area and white area in Figs. 24 and 25 have the same meaning as in Figs. 22 and 23, respectively. Feasible frequency bands in the multipath are decreased compared to those in the LoS path case owing to distortion and power loss by multipath fading.

To show the relationship between $P_{\text {loss }}$ and communication performance, Fig. 26 shows the bit error rate (BER) for $P_{\text {loss }}$. Simulation parameters were as follows. The distance and signalto-noise ratio (SNR) are $10 \mathrm{~cm}$ and $36 \mathrm{~dB}$, respectively. The transmitted signals are Gaussian pulses whose center frequencies are $0.1-9.9 \mathrm{THz}$, and bandwidth is $0.05 \mathrm{THz}$. We assume On-Off keying as the modulation technique. To detect the symbol, an energy detector is utilized [23].

There are two reasons for the increase in the BER. One is the decrease in the received signal power due to molecular absorption and multipath fading. The other is intersymbol interference caused by frequency selectivity [21].

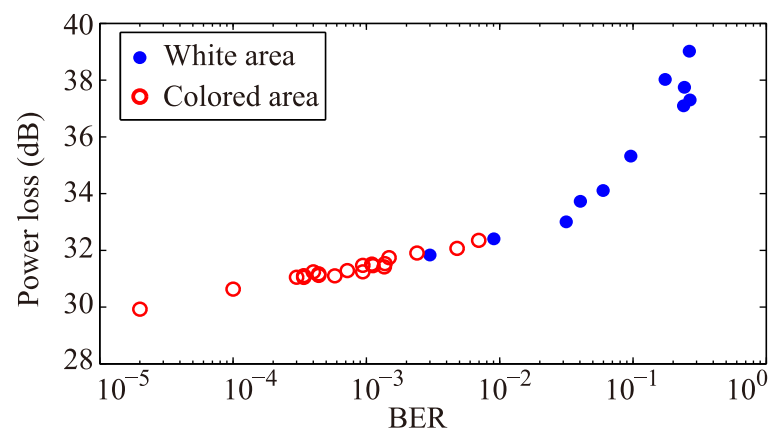

Fig. 26. BER versus channel power loss for $B=0.05 \mathrm{THz}$ at $10 \mathrm{~cm}$.

\section{CONCLUSION}

In this study, we introduced impulse response with minimum phase as a time-domain channel model in the $\mathrm{THz}$ band. We mainly focused on short-range wireless communications, and thus distances less than $100 \mathrm{~cm}$ were considered. We employed the Hilbert transform to obtain the phase component from transmittance containing only the amplitude component. Numerical evaluation showed that the obtained impulse response satisfies causality. In addition, experimental measurements showed the validity of the proposed method.

We also investigated coherence bandwidth of the $\mathrm{THz}$ band in both the LFB and FFB scenarios. Unlike in the UHF band, coherence bandwidth of the $\mathrm{THz}$ band is mostly deterministic as the numerical results showed a strong frequency dependence for the coherence bandwidth. We also showed channel power loss, which represents available power loss with a constraint in terms of target coherence bandwidth. The channel power loss is useful for selecting proper frequency bands for short-range wireless communication.

\section{REFERENCES}

[1] S. Cherry, "Edholm's law of bandwidth," IEEE Spectrum, vol. 41, no. 7, pp. 58-60, Jul. 2004

[2] R. Piesiewicz, C. Jansen, D. Mittleman, T. Kleine-Ostmann, M. Koch, and T. Kurner, "Scattering analysis for the modeling of $\mathrm{THz}$ communication systems," IEEE Trans. Antennas Propag., vol. 55, no. 11, pp. 3002-3009, Nov. 2007.

[3] C. Han, A. O. Bicen, and I. F. Akyildiz, "Multi Ray channel modeling and wideband characterization for wireless communications in the terahertz band," IEEE Trans. Wireless Commun., vol. 14, no. 5, pp. 2402-2412, May 2015.

[4] B. Sklar, "Rayleigh fading channels in mobile digital communication systems part I: Characterization," IEEE Commun. Mag., vol. 35, no. 7, pp. 90-100, Jul. 1997.

[5] J. M. Jornet and I. F. Akyildiz, "Channel modeling and capacity analysis for electromagnetic wireless nanonetworks in the terahertz band," IEEE Trans. Wireless Commun., vol. 10, no. 10, pp. 3211-3221, Oct. 2011.

[6] J. Kokkoniemi, J. Lehtomäki, K. Umebayashi, and M. Juntti, "Frequency and time domain channel models for nanonetworks in terahertz band," IEEE Trans. Antennas Propag., vol. 63, no. 2, pp. 678-691, Feb. 2015.

[7] J. M. Jornet and I. F. Akyildiz, "Femtosecond-long pulse-based modulation for terahertz band communication in nanonetworks," IEEE Trans. Commun., vol. 62, no. 5, pp. 1742-1754, May 2014.

[8] Y. Yang, M. Mandehgar, and D. R. Grischkowsky, "Understanding THz pulse propagation in the atmosphere," IEEE Trans. Terahertz Sci. Technol., vol. 2, no. 4, pp. 406-415, Jul. 2012.

[9] K. Tsujimura, K. Umebayashi, J. Kokkoniemi, and J. Lehtomäki, "A study on channel model for THz band," in Proc. Int. Symp. Antennas Propag.,Okinawa, Japan, Oct. 24-28, 2016, pp. 872-873. 
[10] H. Kuzmany, Solid-State Spectroscopy. New York, NY, USA: SpringerVerlag, 1998.

[11] S. Paine, "The am atmospheric model," Smithsonian Astrophys. Observ., Cambridge, MA, USA, SMA Tech. Memo 152, Feb 2012.

[12] L. S. Rothman et al., "The HITRAN 2008 molecular spectroscopic database," J. Quant. Spectrosc. Radiat. Transf., vol. 110, no. 9-10, pp. 533572, Jun./Jul. 2009.

[13] S. J. Adams, Electromagnetic theory. New York, NY, USA: McGraw-Hill, 1941.

[14] C. Jansen et al., "Diffuse scattering from rough surfaces in THz communication channels," IEEE Trans. Terahertz Sci. Technol., vol. 1, no. 2, pp. 462-472, Nov. 2011.

[15] J. Alan and Z. Daniel, Table of Integrals, Series, and Products, 7th ed. New York, NY, USA: Academic, 2007.

[16] B. Sklar, Digital Communications: Fundamentals and Applications, 2nd ed. Englewood Cliffs, NJ, USA: Prentice-Hall, 2001.

[17] M. Frerking, Digital Signal Processing in Communications Systems. New York, NY, USA: Springer-Verlag, 1993.

[18] J. Watkinson, Convergence in Broadcast and Communications Media, 1st ed. Waltham, MA, USA: Focal Press, 2001.

[19] A. Grami, Introduction to Digital Communications, 1st ed. New York, NY, USA: Academic, 2015.

[20] A. Goldsmith, Wireless Communications. Cambridge, U.K.: Cambridge Univ. Press, 2005.

[21] D. Tse and P. Viswanath, Fundamentals of Wireless Communication. Cambridge, U.K.: Cambridge Univ. Press, 2005.

[22] S. S. Ghassemzadeh, R. Jana, C. W. Rice, W. Turin, and V. Tarokh, "Measurement and modeling of an ultra-wide bandwidth indoor channel," IEEE Trans. Commun., vol. 52, no. 10, pp. 1786-1796, Oct. 2004.

[23] K. Witrisal et al., "Noncoherent ultra-wideband systems," IEEE Signal Process. Mag., vol. 26, no. 4, pp. 48-66, Jul. 2009.

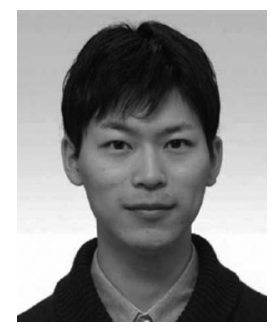

Kazuhiro Tsujimura received the B.E. and M.E degrees in electrical and electronic engineering from the Tokyo University of Agriculture and Technology, Tokyo, Japan, in 2014 and 2016, respectively. He is currently working toward the Ph.D. degree at the Tokyo University of Agriculture and Technology.

From July to September 2015, he was a Visiting Researcher with the Centre for Wireless Communications, University of Oulu, Oulu, Finland. His research interests include wireless communication systems in the THz band.

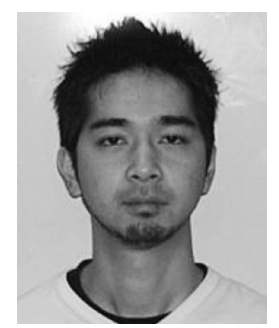

Kenta Umebayashi (S'01-M'04) was born in Tokyo, Japan, in 1974. He received the LL.B. degree from Ritsumeikan University, Kyoto, Japan, in 1996, and the B.E., M.E., and Ph.D. degrees from the Yokohama National University, Yokohama, Japan, in 1999, 2001, and 2004, respectively.

From 2004 to 2006, he was a Research Scientist with the Centre for Wireless Communications, University of Oulu, Oulu, Finland. He is currently an Associate Professor with the Tokyo University of Agriculture and Technology, Tokyo, Japan. He was an Associate Editor of IEICE Transactions on Communications from June 2015 to May 2017. His research interests include signal detection and estimation theories for wireless communication, multiple antenna based signal processing, cognitive radio, and terahertz band wireless communication.

Dr. Umebayashi was the recipient of the Best Paper Award of IEEE WCNC 2012 and the Best Paper Award of the IEEE WCNC Workshop IWSS 2015. He was also the recipient of the IEICE Communication Society Distinguished Service Award in 2010, 2012, and 2017, respectively.

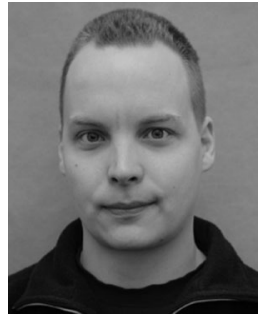

Joonas Kokkoniemi (S'12-M'17) received the B.Sc. (Tech.), M.Sc. (Tech.), and Dr.Sc. (Tech.) degrees in communications engineering from the University of Oulu, Oulu, Finland, in 2011, 2012, and 2017, respectively.

He is currently a Postdoctoral Research Fellow with the Centre for Wireless Communications, University of Oulu. From September to November 2013, he was a Visiting Researcher with the Tokyo University of Agriculture and Technology, Tokyo, Japan. From March to October 2017, he was a Visiting Postdoctoral Researcher with the State University of New York at Buffalo, Buffalo, NY, USA. His research interests include THz band and mmWave channel modeling and communication systems.

Dr. Kokkoniemi was the recipient of several grants and prizes for his work on THz band channel modeling. He served as a Financial Co-Chair for IEICE SmartCom 2016.

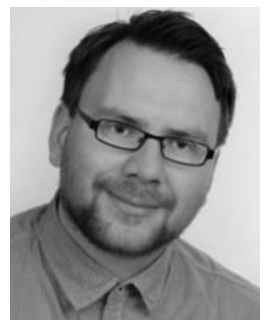

Janne Lehtomäki (S'03-M'06) received the Ph.D. degree from the University of Oulu, Oulu, Finland, in 2005. He was with Georgia Tech, Atlanta, GA, USA, as a Visiting Scholar, in 2013.

$\mathrm{He}$ is currently an Adjunct Professor with the Centre for Wireless Communications, University of Oulu. His current research interest focuses on terahertz band wireless communication.

Dr. Lehtomäki was a co-recipient of the Best Paper Award of IEEE WCNC, 2012. He is an Editorial Board member of Physical Communication. He was a General Co-Chair of the IEEE WCNC 2017 International Workshop on Smart Spectrum, a TPC Co-Chair of the IEEE WCNC 2015 and 2016 International Workshop on Smart Spectrum, and a Publicity/Publications Co-Chair of ACM NANOCOM in 2015, 2016, and 2017. He has served as a Guest Associate Editor for two IEICE Transactions on Communications Special Sections and as a Managing Guest Editor of the Nano Communication Networks Special Issue in 2016

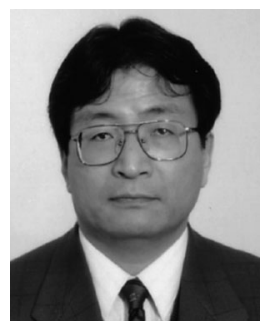

Yasuo Suzuki (M’01) was born in Tokyo, Japan, in August, 1950. He received the B.E. degree from Saitama University, Urawa, Japan, in 1973, and the D.E. degree from the Tokyo Institute of Technology, Tokyo, Japan, in 1985.

In 1973, he joined the Toshiba Corporation, where he worked on the development of various antenna including adaptive antenna for radar, communications, and navigations. In April 2000, he joined the Tokyo University of Agriculture and Technology, where he is currently a Professor with the Department of Electrical and Electronic Engineering. He has experienced a wide range of research and development work such as for array antennas, adaptive antennas, aperture antennas, micro-strip antennas, ultra-compact radio equipment, softwaredefined radio, and so on. He co-authored seven books. His current research interests include the applications of wireless and antenna technologies to mobile communications.

Prof. Suzuki was the recipient of the Paper Award from IEICE 2002. He is an IEICE Fellow. 